\title{
SHORT REPORT \\ Prevalence of Staphylococcus aureus and of methicillin-resistant $S$. aureus clonal complexes in bulk tank milk from dairy cattle herds in Lombardy Region (Northern Italy)
}

\author{
C. CORTIMIGLIA ${ }^{1}$, M. LUINI $^{1}$, V. BIANCHINI ${ }^{1}$, L. MARZAGALLI $^{1}$, \\ F. VEZZOLI ${ }^{1}$, D. AVISANI ${ }^{2}$, M. BERTOLETTI ${ }^{2}$, A. IANZANO $^{3}$, A. FRANCO $^{3}$ \\ AND A. BATTISTI ${ }^{3 *}$ \\ ${ }^{1}$ Istituto Zooprofilattico Sperimentale della Lombardia e dell'Emilia Romagna, Lodi, Italy \\ ${ }^{2}$ Istituto Zooprofilattico Sperimentale della Lombardia e dell'Emilia Romagna, Sorveglianza Epidemiologica \\ Lombardia, Brescia, Italy \\ ${ }^{3}$ Istituto Zooprofilattico Sperimentale del Lazio e della Toscana 'M. Aleandri', Rome, Italy
}

Received 3 November 2015; Final revision 3 May 2016; Accepted 27 June 2016; first published online 26 July 2016

\section{SUMMARY}

Staphylococcus aureus is the most important causative agent of subclinical mastitis in cattle resulting in reduced milk production and quality. Methicillin-resistant S. aureus (MRSA) strains has a clear zoonotic relevance, especially in the case of occupational exposure. The aim of the study was to evaluate the prevalence of $S$. aureus and MRSA in bulk tank milk (BTM) from dairy cattle herds in the Lombardy Region (Northern Italy) and to identify the main MRSA circulating genotypes. MRSA strains were characterized by susceptibility testing, multi-locus sequence typing (MLST), spa typing and SCCmec typing. A total 844 BTM samples were analysed and S. aureus and MRSA were detected in $47 \cdot 2 \%$ and $3.8 \%$ of dairy herds, respectively. MLST showed that the majority (28/32) of isolates belonged to the typical livestock-associated lineages: ST398, ST97 and ST1. Interestingly, in this study we report for the first time the new ST3211, a single locus variant of ST(CC)22, with the newly described 462 aroE allele. Our study indicates high diffusion of $S$. aureus mastitis and low, but not negligible, prevalence of MRSA in the considered area, suggesting the need for planning specific control programmes for bovine mastitis caused by $S$. aureus, especially when MRSA is implicated.

Key words: Bulk tank milk, dairy cattle, genotyping, MRSA, Staphylococcus aureus.

Staphylococcus aureus is one of the most important causes of contagious and chronic mastitis in dairy cattle, leading to significant economic losses in dairy industry. The presence of $S$. aureus in raw milk also represents a potential source for the introduction of the pathogen into the dairy food chain, with a consequent risk of food contamination [1].

\footnotetext{
* Author for correspondence: Dr A. Battisti, Istituto Zooprofilattico Sperimentale del Lazio e della Toscana, Via Appia Nuova 1411, Rome 00178, Italy.

(Email: antonio.battisti@izslt.it)
}

Methicillin-resistant S. aureus (MRSA) represents a relevant issue for human health and over the last 10 years it has been recognized as a frequent colonizer of different animal species, including pigs, cattle, horses, and poultry. The resistance mechanism is due to the acquisition of gene mec $\mathrm{A}$ or its homologue $m e c \mathrm{C}$, harboured by different types of staphylococcal cassette chromosome mec (SCCmec). In Italy, livestockassociated (LA)-MRSA from food-producing animals mainly belongs to clonal complex (CC) 398 , although other clones, such as $\mathrm{CC} 97$ and $\mathrm{CC} 1$, are widespread $[2,3]$. 
The detection of MRSA in bovine milk and dairy cattle herds is increasingly reported worldwide, although with low prevalence, with the majority of strains belonging to sequence type (ST) 398 [4]. The emergence of MRSA in cattle complicates the treatment of bovine mastitis caused by $S$. aureus for its frequent multidrug-resistant (MDR) nature, while its zoonotic potential to farmers, farm workers and veterinarians is of concern for public health [1]. Therefore, our work was aimed at estimating the prevalence of $S$. aureus and MRSA in bulk tank milk (BTM), providing phenotypic and molecular characterization of MRSA isolates for further insight into lineages circulating in dairy cattle herds in the Lombardy Region (Northern Italy).

Milk samples were collected between July 2012 and October 2013 from the bulk tank of 844 out of 7008 dairy cattle farms of the Lombardy Region, distributed in 11 provinces. The sample size was determined based on $95 \%$ confidence and an expected prevalence of MRSA of $7 \%$ from unpublished data, accounting the number of herds per province. Average herd size and milk production in the area were 190 and 9000 $\mathrm{kg} /$ year, respectively. For each selected farm, data about the presence of other animals, including noncommercial livestock, (pigs, horses, poultry, small ruminants) were collected by consulting the Regional Register for Livestock.

Milk aliquots from the enrolled herds were obtained by local veterinary authorities, which submitted samples as a part of their routine monitoring programmes. The samples were transported chilled to the laboratory in sterile tubes containing sodium azide $(0 \cdot 12 \mu \mathrm{g} / \mathrm{ml})$ and stored at $-20{ }^{\circ} \mathrm{C}$ until analysed. Bacteriological and molecular diagnosis were performed as described previously [3]. After thawing, milk samples were shaken to disperse fat globules. The samples were diluted $1: 10$ in $0 \cdot 1 \%$ sterile peptone water and $100 \mu \mathrm{l}$ of the milk and of the 1:10 dilution were spread on Baird-Parker agar supplemented with rabbit plasma fibrinogen (BP-RPF; Oxoid, UK), incubated at $37^{\circ} \mathrm{C}$ for $48 \mathrm{~h}$. Colonies developing a typical coagulase halo were presumptively considered as $S$. aureus according to the ISO 6888-2:1999 'Horizontal method for the enumeration of coagulasepositive staphylococci (Staphylococcus aureus and other species): technique using rabbit plasma fibrinogen agar medium'. The number of colony-forming units (c.f.u.)/ml was calculated as the weighted average of the number of typical colonies on two BP-RPF plates. Four colonies of S. aureus from each positive sample were screened for methicillin susceptibility by disk diffusion test with $30 \mu \mathrm{g}$ cefoxitin on Mueller-Hinton agar plates (both from Oxoid), performed and interpreted according to CLSI Standard M100 S23. Resistant strains were subjected to a multiplex polymerase chain reaction (PCR) targeting the $S$. aureus-specific nuc gene and the mecA gene and to a PCR targeting the $m e c \mathrm{C}$ gene. To estimate the c.f.u./ml for MRSA, according to ISO 7218:2007E 'Method of calculation: after identification', we first counted the number of the screened colonies confirmed as mecA- or mecC-positive $S$. aureus out of the four colonies tested, and then we reported the MRSA c.f.u./ml count as the proportion of MRSA-confirmed colonies on the total number of $S$. aureus colonies found on the BP-RPF agar plate. In addition to direct plating, $3 \mathrm{ml}$ milk were enriched in Mueller-Hinton broth (Oxoid) with $7 \cdot 5 \% \mathrm{NaCl}$ for $24 \mathrm{~h}$ at $37^{\circ} \mathrm{C}$, followed by selective enrichment in tryptic soy broth (Oxoid) supplemented with $5 \mathrm{mg} / 1$ oxacillin. After $24 \mathrm{~h}$ incubation at $37^{\circ} \mathrm{C}$, a loopful of the enrichment broth was plated on Brilliance ${ }^{\mathrm{TM}}$ MRSA 2 agar (Oxoid) and then incubated for a further $24 \mathrm{~h}$. Presumptive MRSA colonies grown on Brilliance MRSA 2 agar were subcultured and confirmed by the two above-mentioned PCR methods.

MRSA strains were tested for the susceptibility to antimicrobials using the broth microdilution method, following EUCAST (www.eucast.org) recommendations, with data interpreted according to EUCAST epidemiological cut-offs. Eighteen antimicrobial agents were tested: clindamycin, erythromycin, tiamulin, tetracycline, ciprofloxacin, rifampicin, cefoxitin, streptomycin, kanamycin, linezolid, fusidic acid, mupirocin, penicillin, vancomycin, sulfonamide, chloramphenicol, gentamicin and trimethoprim. The determination of inducible clindamycin resistance was performed according to the CLSI standard.

MRSA strains were genotyped by multi-locus sequence typing (MLST), spa typing, and SCCmec typing and subtyping, according to previously reported methods [2].

Statistical analysis was performed using R v. 3.1.2 (https://cran.r-project.org/bin/windows/base/old/3.1.2/) and SAS v. 9.1 (SAS Institute Inc., USA). The herd size of $S$. aureus or MRSA-positive and -negative farms was compared using a non-parametric test for independent samples (one-tailed Wilcoxon-MannWhitney test). Pearson's $\chi^{2}$ test was applied in order to assess the independence between the presence of 
S. aureus or MRSA in BTM and presence of other animals (pigs, horses, poultry, small ruminants). Fisher's exact test was applied when expected frequencies were $<5$.

S. aureus was isolated from 398 of the 844 BTM samples $[47 \cdot 2 \%, 95 \%$ confidence interval (CI) $43 \cdot 8$ $50 \cdot 5]$, with prevalence in different provinces ranging from $29 \cdot 2 \%$ to $68 \cdot 5 \%$ (Table 1). MRSA was found in 32 out of 844 BTM samples $(3 \cdot 8 \%, 95 \%$ CI $2 \cdot 5-5 \cdot 1)$. Fifteen out of 32 strains were isolated by direct plating, 11 were detected following enrichment, and the remaining six samples tested positive for MRSA by both methods. In all cases, resistance was conferred by the mecA gene, while mec $\mathrm{C}$ was never detected. Prevalence by province varied between $0.0 \%$ (Como and Lecco) and $10 \cdot 8 \%$ (Cremona) (Table 1). MRSA counts were $<10$ c.f.u./ml in 11 out of 32 samples (detected only following enrichment). In the remaining 21 samples counts were between $10^{1}$ to $5 \times 10^{2}$ c.f.u. $/ \mathrm{ml}$.

Susceptibility testing revealed the presence of 27 different resistance patterns (Supplementary Table S1). In addition to cefoxitin and beta-lactams, most of the strains were resistant to tetracycline (30/32, $93 \cdot 7 \%)$. Most of the isolates $(29 / 32,90 \cdot 6 \%)$ were also MDR (i.e. to at least three antimicrobial classes/subclasses) and 20 strains $(62 \cdot 5 \%)$ showed a pattern of resistance to $\geqslant 5$ antimicrobials. A total of 13 isolates $(40 \cdot 6 \%)$ were microbiologically and clinically resistant to fluoroquinolones (CIP MIC $>1 \mathrm{mg} / \mathrm{l}$, range 2-16 $\mathrm{mg} / \mathrm{l})$, with $>50 \%$ (7/13) belonging to $\mathrm{ST}(\mathrm{CC}) 97$. High rates of macrolide-lincosamide resistance were observed, with $43 \cdot 7 \%$ to erythromycin (14/32), and $46.9 \%$ to clindamycin (15/32); the latter was confirmed to be inducible resistance in six isolates $(n=5 \mathrm{ST} 1, \mathrm{spa}$ type t127; $n=1$ ST5, spa type t535; Supplementary Table S1). Of the aminoglycosides tested, kanamycin resistance was the most frequent, accounting for $40 \cdot 6 \%(13 / 32)$ of isolates. Diaminopyrimidine resistance (trimethoprim) reached $28 \cdot 1 \%$, mostly observed in ST398 isolates.

A total of six different STs were identified, of which ST398 $(n=13)$, ST1 $(n=7)$ and ST97 $(n=7)$ were the most frequently observed (Table 2). A single CC22 isolate (spa type t309), proved to be a new single locus variant (SLV) (in the aro $\mathrm{E}$ gene, allele 462) of ST22, filed as ST3211 by the curator of the $S$. aureus MLST database (http://saureus.mlst.net/sql/sthtml. asp). Thirteen spa types were found, with t899/ ST398 $(n=9)$ and t127/ST1 $(n=7)$ representing the most dominant. All strains harboured SCCmec cassette types IV or V.
The relationship between the number of $S$. aureuspositive herds and herd size was investigated excluding five cattle herds for which it was not possible to trace back the number of heads. The median value of herd size for $S$. aureus-positive and $S$. aureus-negative farms was 102 and 145 heads, respectively. This difference turned out to be statistically significant $(P=$ 0.003). The same analysis applied to MRSA, revealed a statistically smaller herd size in negative (median value 123) than in positive (median value 291) farms $(P<0.001)$. Statistical analysis did not show any significant association between the presence of pigs or other farm animals and presence of $S$. aureus and MRSA in these herds $(P>0.05)$ (Supplementary Table S2).

Our study demonstrated high prevalence of $S$. aureus in bovine bulk milk (47.2\%) in a high-density dairy farming area like Lombardy Region, where $>40 \%$ of Italian dairy milk is produced. This finding is in agreement with other studies reporting high prevalences in other European and North-American countries [5, 6].

MRSA has been identified as an emerging pathogen in livestock and in the last 10 years, the detection of MRSA in cases of udder infection has been increasingly reported [4], but few studies have evaluated the prevalence of MRSA in BTM samples. Prevalence of $1.3 \%$ was reported by Haran et al. [5] in the United States, while in Germany, Kreausukon and co-workers [7] found MRSA strains in $4.4 \%$ of the analysed BTM samples. In line with previous findings, in the present study the prevalence of MRSA in BTM resulted in a low, but not negligible (3.8\%) value. In addition, our study showed low or very low MRSA BTM contamination in positive herds (from $<10$ herds, but isolated only by enrichment to $5 \times 10^{2}$ c.f.u./ml), suggesting low or very low within-herd prevalence of intra-mammary infection (IMI) caused by MRSA and/or a low excretion of MRSA by infected cows. This observation is in agreement with other findings reported recently in Italy for IMI in dairy cattle [8]. S. aureus and MRSA counts may have been slightly affected by some limitations to our study. First, samples contained sodium azide, a preservative used especially in the case of somatic cell count quantification or fat and protein analysis. Nevertheless $S$. aureus has a high degree of tolerance to this compound which is allowed in raw milk in order to conserve the bacteriological characteristics at low temperature during transportation [9]. Moreover, samples were stored at $-20^{\circ} \mathrm{C}$ until analysis. Nevertheless, it is well known that occurrence of $S$. aureus in milk 
Table 1. Prevalence of $\mathrm{S}$. aureus and MRSA in bulk tank milk of the 11 provinces and associated confidence intervals

\begin{tabular}{|c|c|c|c|c|c|c|}
\hline \multirow[b]{2}{*}{ Province } & \multirow[b]{2}{*}{ Median herd size } & \multirow[b]{2}{*}{ Sampled farms } & \multicolumn{2}{|l|}{ S. aureus } & \multicolumn{2}{|l|}{ MRSA } \\
\hline & & & Positive samples & $\%(95 \% \mathrm{CI})^{*}$ & Positive samples & $\%(95 \% \mathrm{CI}) \dagger$ \\
\hline Bergamo & 73 & 91 & 53 & $58 \cdot 2(48 \cdot 1-68 \cdot 4)$ & 3 & $3 \cdot 3(0 \cdot 7-9 \cdot 1)$ \\
\hline Brescia & 149 & 97 & 53 & $54 \cdot 6(44 \cdot 2-64 \cdot 7)$ & 1 & $1 \cdot 0(0-5 \cdot 6)$ \\
\hline Como & 57 & 65 & 19 & $29 \cdot 2(18 \cdot 9-42 \cdot 0)$ & 0 & $0(0-5 \cdot 5)$ \\
\hline Cremona & 328 & 91 & 42 & $46 \cdot 2(39 \cdot 4-55 \cdot 8)$ & 10 & $10 \cdot 8(5 \cdot 3-10 \cdot 9)$ \\
\hline Lecco & 51 & 30 & 13 & $43 \cdot 3(26 \cdot 0-62 \cdot 3)$ & 0 & $0(0-11 \cdot 6)$ \\
\hline Lodi & 282 & 78 & 35 & $44 \cdot 9(33 \cdot 7-56 \cdot 5)$ & 5 & $6 \cdot 4(2 \cdot 1-14 \cdot 3)$ \\
\hline Mantova & 167 & 92 & 43 & $35 \cdot 8(26 \cdot 7-45 \cdot 0)$ & 2 & $5 \cdot 7(2 \cdot 1-11 \cdot 9)$ \\
\hline Milano & 155 & 106 & 38 & $46 \cdot 7(35 \cdot 5-56 \cdot 3)$ & 6 & $2 \cdot 1(0 \cdot 3-7 \cdot 5)$ \\
\hline Pavia & 173 & 54 & 18 & $33 \cdot 3(21 \cdot 5-47 \cdot 6)$ & 2 & $3 \cdot 7(0 \cdot 5-12 \cdot 8)$ \\
\hline Sondrio & 20 & 92 & 63 & $68 \cdot 5(57 \cdot 8-77 \cdot 5)$ & 2 & $2 \cdot 2(0 \cdot 3-7 \cdot 6)$ \\
\hline Varese & 93 & 48 & 21 & $43 \cdot 8(29 \cdot 8-58 \cdot 7)$ & 1 & $2 \cdot 1(0-11 \cdot 1)$ \\
\hline Total & 127 & 844 & 398 & $47 \cdot 2(43 \cdot 8-50 \cdot 5)$ & 32 & $3 \cdot 8(2 \cdot 5-5 \cdot 1)$ \\
\hline
\end{tabular}

* Confidence intervals based on the Normal distribution approximation.

$\dagger$ Exact confidence intervals based on Clopper-Pearson's method.

Table 2. Genotypic characteristics of the 32 MRSA isolates detected from bulk tank milk

\begin{tabular}{llll}
\hline \hline CC & ST & $\begin{array}{l}\text { No. of } \\
\text { strains }\end{array}$ & spa type/SCCmec type $(n)$ \\
\hline 398 & 398 & 14 & t899/V (7), t899/IVb (2), t034/V (2), \\
& & & t011/V (2), t108/V (1) \\
97 & 97 & 7 & t1730/V (3), t4795/V (2), t2421/V (1), \\
& & & t9295/V (1) \\
1 & 1 & 7 & t127/IVa (7) \\
5 & 5 & 2 & t688/V (1), t548/V (1) \\
5 & 461 & 1 & t309/IV (1) \\
22 & 3211 & 1 &
\end{tabular}

CC, Clonal complex; ST, sequence type.

samples does not significantly change after thawing. Finally, discordant results between direct cultures and cultures after enrichment occurred. This may have been partly due to the high selectivity of the two enrichment steps. For instance, the $7 \cdot 5 \%$ salt concentration in the enrichment broth, may not have favoured the growth of MRSA compared to other salt-tolerant bacteria, including clones of MSSA. However, parallel testing (i.e. direct cultures and enrichment cultures) is likely to have kept the overall sensitivity of the diagnostic test adequate.

Between-province variation in the prevalence of $S$. aureus and MRSA was observed and may be explained by differences in farming practices. For example, the highest prevalence of $S$. aureus was detected in Sondrio province, where farms are usually small (median value 20) and managed by grazing on mountain pastures. These conditions allow contacts between animals from different farms and may promote the spread of infection. The reason why larger farms were more likely to be MRSA-positive than smaller ones is not known. A possible explanation is that larger farms are more likely to use more antimicrobials (i.e. for routine dry-off therapy or in the case of a rise in somatic cell count) or to introduce animals from other intensive dairies and are thereby more likely to be affected by or exposed to MRSA clones. Indeed, higher levels of trading contacts were suggested among predictors for the positive significant association between larger farm size and presence of MRSA, as demonstrated in the Italian pig industry [2]. However, the analytical sensitivity of the method and the effects of larger numbers of animals screened by the BTM test on diagnostic sensitivity at aggregate level may have increased the probabilities of a positive outcome of the assay in larger herds.

In this study, the presence of pigs or other farm animals was considered and investigated as a potential risk factor, but no association was found between $S$. aureus or MRSA-positive status in BTM and the presence of any of the animal species in the same farm (Supplementary Table S2).

The results of this study demonstrate a high level of multidrug-resistance in MRSA isolates in dairy farms in Northern Italy, which has already been observed in MRSA clonal complexes from Italian primary productions [2]. Selection pressure by the use of tetracyclines has been considered among major causes for the spread of CC398 S. aureus in food-producing 
animals in four continents [1], even before acquisition of the mecA gene through the SCCmec cassette. It has already been documented that LA-MRSA strains isolated from bovine milk $[10,11]$ as well as all $\beta$-lactams are frequently co-resistant to tetracyclines, macrolides, lincosamides and aminoglycosides. This is of further concern, as most of these antimicrobials are also employed for the treatment of bovine mastitis and in general for antimicrobial therapy in dairy cattle. Moreover, our data also show that $>60 \%$ of the isolates were resistant to $\geqslant 5$ antimicrobial classes, confirming the MDR nature of MRSA clones in Northern Italy.

With regard to molecular typing, a large proportion of the isolates belong to the typical LA-MRSA CC398, the most prevalent LA-MRSA in Europe [1]. Of ST398 isolates, four different spa types were identified, with 1899 representing the dominant type [2]. As regards the other LA-MRSA lineages detected, CC1, spa type 127 was frequently reported in Italy in pigs, cattle and humans, and represents another lineage of concern among LA-MRSA colonizing pigs and dairy cattle [10]. Similarly, CC97 is known to be one of the $S$. aureus lineages associated with cattle, and a CC97 MRSA clone is one of the most important lineages in pigs and cattle in Italy [2, 11]. Consistent with previous findings [10,11], the typing of the SCCmec elements in LA-MRSA lineages shows the presence of types IV and V only.

Strains belonging to CC5 and CC22, typical human MRSA also reported in Italy [12], were also isolated and their presence in BTM samples may be due to either colonization of dairy cattle or to human direct or indirect contamination of BTM. Interestingly, in this study we report first on the new ST3211, a SLV of ST(CC)22, with the newly described 462 aroE allele. Some characteristics of this isolate (e.g. SCCmec IV, ciprofloxacin resistance, gentamycin susceptibility) are consistent with those described for the successful ST22 human clone in Italy [12].

Emergence of MRSA in cattle poses a potential risk for animal-to-human direct transmission and for human colonization via the consumption of raw milk or unpasteurized dairy products, although no conclusive evidence is available at present. Indeed, it is well known that people with close contact with LA-MRSA carrier animals are at risk of being colonized and infected by these lineages [1], considering the high genetic relatedness between some Italian LA-MRSA strains from dairy cattle and humans [10].
In conclusion, the high prevalence of $S$. aureus in BTM underlines the importance of this bacterial pathogen as a cause of mastitis in dairy herds of an intensive production area such as the Lombardy Region. Similarly, a lower, but not negligible, prevalence of MRSA is of note because the diffusion of such strains may be enhanced by inappropriate or empirical treatment with antibiotics administered without any prior susceptibility testing. Also of concern are the risks associated with occupational exposure for milkers and other farm workers, while the evidence of MRSA infection caused by contaminated milk or dairy products needs to be thoroughly assessed. The generally low MRSA counts found in BTM is reasonably due to a low number of infected cows and/or to a low shedding of MRSA by infected cows [8], and merits further investigation, since it may influence the magnitude of occupational exposure. However, as bacteriological investigations are usually performed only in case of serious problems of mastitis, it is also likely that subclinical MRSA IMI infections remain undetected and so far underestimated. Thus, microbiological investigations of milk quality in cattle should be performed on a routine basis, including susceptibility testing, for the screening of zoonotic and MDR pathogens. Additionally, more stringent control measures against $S$. aureus mastitis in cattle are advisable, with severe decision criteria on animals positive for MRSA. In this respect, special attention should be paid to all management practices capable of minimizing the risks of MRSA colonization, infection and transmission in the intensive dairy cattle farming system.

\section{SUPPLEMENTARY MATERIAL}

For supplementary material accompanying this paper visit http://dx.doi.org/10.1017/S0950268816001576.

\section{ACKNOWLEDGEMENTS}

The authors thank Francesca Pozzi, Patricia Alba, Roberta Amoruso for their skilled technical assistance. This work was funded by the Italian Ministry of Health - Ricerca Corrente IZSLER 2010/03.

\section{DECLARATION OF INTEREST}

None. 


\section{REFERENCES}

1. Guardabassi L, et al. Public health impact and antimicrobial selection of methicillin-resistant staphylococci in animals. Journal of Global Antimicrobial Resistance 2013; 1: 55-62.

2. Battisti A, et al. Heterogeneity among methicillinresistant Staphylococcus aureus from Italian pig finishing holdings. Veterinary Microbiology 2010; 142: 361-366.

3. Cortimiglia C, et al. Short communication: Prevalence of Staphylococcus aureus and methicillin-resistant $S$. aureus (MRSA) in bulk tank milk of dairy goat farms from Northern Italy. Journal of Dairy Science 2015; 98: 2307-2311.

4. Holmes MA, Zadoks RN. Methicillin resistant S. aureus in human and bovine mastitis. Journal of Mammary Gland Biology and Neoplasia 2011; 16: 373-382.

5. Haran KP, et al. Prevalence and characterization of Staphylococcus aureus, including methicillin-resistant Staphylococcus aureus, isolated from bulk tank milk from Minnesota dairy farms. Journal of Clinical Microbiology 2012; 50: 688-695.

6. Jørgensen HJ, et al. Enterotoxigenic Staphylococcus aureus in bulk milk in Norway. Journal of Applied Microbiology 2005; 99: 158-166.
7. Kreausukon K, et al. Prevalence, antimicrobial resistance, and molecular characterization of methicillinresistant Staphylococcus aureus from bulk tank milk of dairy herds. Journal of Dairy Science 2012; 95: 4382-4388.

8. Luini M, et al. Methicillin-resistant Staphylococcusaureus (MRSA) is associated with low within-herd prevalence of intra-mammary infections in dairy cows: genotyping of isolates. Veterinary Microbiology 2015; 178: 270-274.

9. Silcock P, et al. Microbially induced changes in the volatile constituents of fresh chilled pasteurised milk during storage. Food Packaging and Shelf Life 2014; 2: 81-90.

10. Alba $\mathbf{P}$, et al. Livestock-associated methicillin resistant and methicillin susceptible Staphylococcus aureus sequence type (CC)1 in European farmed animals: high genetic relatedness of isolates from Italian cattle herds and humans. PLoS ONE 2015; 10: e0137143.

11. Feltrin F, et al. A livestock-associated, multidrugresistant, methicillin-resistant Staphylococcus aureus clonal complex 97 lineage spreading in dairy cattle and pigs in Italy. Applied and Environmental Microbiology 2015; 82: 816-821.

12. Baldan R, et al. Epidemic MRSA clone ST22-IV is more resistant to multiple host- and environment-related stresses compared with ST228-I. Journal of Antimicrobial Chemotherapy 2015; 70: 757-765. 